\title{
Stroke severity predicted by aortic atheroma detected by ultra-fast and cardiac-gated chest tomography ${ }^{\dagger}$
}

\section{Marc A. Lazzaro ${ }^{1}$, Osama O. Zaidat ${ }^{1,2,3 *}$, Mohammad A. Issa ${ }^{1}$, Robert C. Gilkeson ${ }^{4}$, Jeffrey L. Sunshine ${ }^{4}$, Robert W. Tarr ${ }^{2,3}$, Shakir Husain ${ }^{5 *}$ and Jose I. Suarez ${ }^{6}$}

1 Department of Neurology, Medical College of Wisconsin/Froedtert Hospital, Milwaukee, WI, USA

${ }^{2}$ Department of Neurosurgery, Medical College of Wisconsin/Froedtert Hospital, Milwaukee, WI, USA

${ }^{3}$ Department of Radiology, Medical College of Wisconsin/Froedtert Hospital, Milwaukee, WI, USA

${ }^{4}$ Department of Radiology, Case Medical School, Cleveland, OH, USA

${ }^{5}$ Department of Neurology, MAX Institute of Neurosciences, New Delhi, India

${ }^{6}$ Department of Neurology, Baylor College of Medicine, Houston, TX, USA

\section{Edited by:}

Afshin A. Divani, University of

Minnesota, USA

Reviewed by:

Afshin Borhani Haghighi, Saint Louis University, USA

Afshin A. Divani, University of

Minnesota, USA

*Correspondence:

Osama O. Zaidat, Department of

Neurology, Neurosurgery and

Radiology, Medical College of

Wisconsin/Froedtert Hospital,

Milwaukee, WI 53226, USA.

e-mail: szaidat@mcw.edu;

Shakir Husain, Department of

Neurology, MAX Institute of

Neurosciences, 2 Press Enclave

Marg, Saket, New Delhi 110017, India.

e-mail:drshakir@gmail.com

\begin{abstract}
Background and Purpose: The presence of aortic atherosclerosis is an independent risk factor for secondary stroke. The present study was designed to have an initial exploration of the correlation between the load and extent of aortic atheroma (AA) and initial stroke severity or clinical outcome 3 months after stroke. Methods: Cardiac-gated chest tomography (CGCT) was used to detect and measure AA in patients with acute ischemic stroke as shown by our group in prior prospective studies and this is part four sub-exploratory study of the same cohort. The National Institute of Health Stroke Scale (NIHSS) was used to assess the initial stroke severity, and the modified Rankin Scale (mRS) was used to assess 3-month outcome. Results: Thirty-two patients underwent CGCT for evaluation of AA, and 21 were found to have AA. AA was more prevalent in patient with NIHSS $>6(14 / 17$ versus $7 / 15, p$-value 0.03 ). Applying the multiple logistic regression and propensity score adjustment (using the propensity of having AA given the baseline features as covariates) showed a non-significant trend that AA is three times more likely to be associated with NIHSS $>6$ $(p=0.08$, OR $3.08,95 \% \mathrm{Cl} 0.94-13.52)$. There was no evidence of association of AA with 3-month functional outcome (mRS): 11/14 (78.6\%) mRS >1 had AA, and 10/18 (55.5\%) of those with $m R S \leq 1$ had AA $(p=0.27)$. Conclusion: In our current study with limited sample number and exploratory nature, the presence of AA on CGCT with acute ischemic stroke patients may be associated with worse neurological deficit at presentation. There was no evidence of association with 3-month functional outcome using the mRS.
\end{abstract}

Keywords: stroke, outcome, aortic atheroma, cardiac-gated CT, TEE, stroke severity, atherosclerosis

\section{INTRODUCTION}

Atherosclerosis in the ascending or the proximal thoracic aorta is an independent risk factor for secondary stroke (Acarturk et al., 1995; Jones et al., 1995; Cardenas, 1996; The French Study of Aortic Plaques in Stroke Group, 1996; Cohen et al., 1997a,b; Castellanos et al., 2001; Amanuallah et al., 2002; Zaidat et al., 2002a) The risk is related to the shape and characteristics of the aortic plaque and its thickness as measured in millimeters (mm) (Jones et al., 1995; The French Study of Aortic Plaques in Stroke Group, 1996; Zaidat et al., 2002a) The estimated relative risk of future stroke in patients with more than $4 \mathrm{~mm}$ aortic atheroma (AA) ranges between 1.6 and 4.3 (Acarturk et al., 1995; Jones et al., 1995; Cardenas, 1996; The French Study of Aortic Plaques in Stroke Group, 1996; Cohen et al., 1997a; Castellanos et al., 2001; Amanuallah et al., 2002; Zaidat et al., 2002a) The aim of this study is to provide exploratory data on whether the initial stroke severity and/or the clinical 3-month disability outcome can be correlated with the presence or absence of AA as detected on cardiac-gated chest tomography (CGCT).

${ }^{\dagger}$ Presented in the 55th Annual American Academy of Neurology Meeting.
We have shown in our prior studies that the newer and faster multi-slice CT scanners and techniques provide reliable information about AA in patients presenting with acute ischemic stroke when compared to transesophageal echocardiography (TEE) and as part of the same project we prospectively designed this current study to correlate the presence of AA with stroke severity and 3-month clinical outcome (Costello et al., 1992; Chung et al., 1996; Fuchs et al., 2000; Flohr et al., 2002; Roos et al., 2002; Zaidat et al., 2002b, 2003). CGCT is cardiac cycle gated (to limit the motion artifact) and ultrafast (only a single breath hold to complete the actual study). CGCT was found to delineate AA features and detect smaller plaques in higher proportions of patients than TEE (Costello et al., 1992; Acarturk et al., 1995; Cardenas, 1996; Chung et al., 1996; Cohen et al., 1997a,b; Castellanos et al., 2001; Amanuallah et al., 2002; Roos et al., 2002; Zaidat et al., 2002a,b) thus it was chosen to evaluate for AA.

\section{MATERIALS AND METHODS}

Patients with acute ischemic stroke, who were admitted to University Hospital of Cleveland, a tertiary medical center, between February and November of 2000 were identified. Of this cohort, 
patients who underwent CGCT to evaluate AA were selected. Over the interval of the study, there were no consistent guidelines for the use of CGCT in looking for the etiology of ischemic stroke. The CGCT procedure was selected for the patients who had technically inadequate TEE studies, or for those in whom TEE had been unrevealing. To assess for a selection bias, the study sample was compared to the remaining patients with TEE procedures who did not have CGCT.

\section{SELECTION CRITERIA}

(1) Adult 18 years or older.

(2) Acute ischemic stroke diagnosed by a stroke neurologist.

(3) Completed CGCT for the purpose of evaluating AA.

\section{EXCLUSION CRITERIA}

(1) Stroke patients who did not undergo the CGCT.

(2) Patients who could not complete the CGCT.

(3) The image quality was not adequate for reliable interpretation.

\section{STUDY OUTCOMES}

The explanatory variable was the presence or absence of AA on the CGCT and the response variables were stroke severity at presentation and 3-month outcome. The initial stroke severity was assessed using the National Institute of Health Stroke Severity Scale (NIHSS). The NIHSS is routinely recorded in ischemic stroke patients admitted to our institution. The NIHSS was dichotomized to less or equal to 6 points for mild and moderate to severe stroke.

The second clinical outcome of interest is the 3-month modified Rankin disability scale (modified Rankin Scale, mRS). The mRS ranges between 0 and 6 , where 0 represents normal function without any residual deficit following a stroke and 6 indicates death. The mRS was dichotomized to $0-1$ and 2-6 for good and bad outcomes, respectively.

\section{STATISTICAL ANALYSIS}

The statistical analyses were performed using SPSS V11.0.1 (2001, SPSS Inc.), JMP V3 (SAS Institute, Cary, NC, USA, 1995), and Analyse-it (by Analyse-It Software Inc., England, UK, 2002).

\section{DESCRIPTIVE STATISTICS}

Demographics, clinical, and imaging baseline data were presented in a table format, giving the mean with standard deviations $(M \pm \mathrm{SD})$ and median with inter-quartile ranges. For categorical variables, frequencies, proportions, and percentages were provided. Student's two sample $t$-tests were used to compare the continuous normally distributed data with unequal variances. Fisher's exact test and chi-square were used to compare the proportions among the binary variables. Level of significance was selected at the conventional and standard type I error of $\alpha \leq 0.05$.

\section{OUTCOME ANALYSIS}

Associations between atheroma presence and stroke severity as estimated by NIHSS, or of atheroma presence and 3-month mRS were examined using multiple logistic regression to adjust for other known stroke risk factors, including: age, sex, race, hypertension (HTN), diabetes (DM), smoking, and coronary artery disease (CAD). To allow adjustment for other covariates, several models were built with up to five covariates in each model in accordance with parsimonies rule. Factors known to be associated with stroke severity at presentation were included to adjust for confounders or effect modifiers. Prior to incorporating these factors into the model, a univariate analysis was performed to asses the relation of the baseline variables and AA (independent variable) to initial Stroke Severity (response variable).

Variables were incorporated in the model if they were deemed clinically significant or had a $p$-value of $<0.5$ on the univariate analysis. We allowed for a large $p$-value because of the sample size and the nature of the exploratory analysis. Regression coefficients were estimated using the maximum likelihood. Goodness of fit was assessed using the likelihood ratio test with chi-square statistics between the full and reduced models. Co-linearity between covariates were assessed by scatter plots and comparing the regression models with and without the variable of interest.

Due to the small sample size, there was limited ability to adjust for other covariates simultaneously using the standard logistic regression, and propensity score (PS) analysis was used to confirm the findings. The PS technique was implemented to incorporate all baseline variables into one continuous score based on the probability of having AA. The PS is the probability between 0 and 1 for a subject to have atheroma present given all his baseline variables, and is estimated using standard logistic regression. The PS model was evaluated for its ability to discriminate between subjects who had atheromas and those who did not, using the area under the receiver operating characteristic (ROC) curve (C Statistic). After calculating the probability for every patient, the PS was incorporated in the regression model as a continuous covariate in addition to the AA to adjust for confounders (Joffe and Rosenbaum, 1999).

\section{RESULTS}

A total of 32 patients were found to have undergone CGCT for the purpose of evaluating stroke etiology.

\section{AORTIC ATHEROMA AND INITIAL STROKE SEVERITY}

There were 21 patients with AA on CGCT, 7 with NIHSS $\leq 6$ and 14 with NIHSS $>6(p=0.03)$. The univariate analysis revealed association of these baseline variables with a $p$-value of less than 0.5 (a larger $p$-value was used due to the small sample size) when analyzed against NIHSS: age, ethnicity, HTN, DM, CAD, hyperlipidemia, AA thickness, and smoking (see Table 1). CAD, AA thickness, large atheroma $>4 \mathrm{~mm}$ (consistent with the standard in the literature of what is thought to be significant atheroma thickness), and atheroma presence were associated with worse initial stroke on univariate analysis ( $p$-value $<0.05$ ). Multiple logistic regression with NIHSS $>6$ as the response variable and looking at the effect of age, CAD, presence or absence of AA, and PS (weighted variable of baseline characteristics according to univariate result) was performed. This multivariate analysis model showed that AA has a slight trend, but no statistical significance, with ischemic stroke presenting with an initial NIHSS $>6(p=0.08$, OR 3.08, 95\% CI 0.94-13.52), as shown in Table 2.

\section{AORTIC ATHEROMA AND 3-MONTH CLINICAL STROKE OUTCOME}

On the univariate analyses correlating AA with 3-month disability outcome as measured by $\mathrm{mRS}$; the following variables had 
Table 1| Univariate analysis of the baseline features and initial stroke severity ( $n, \%$, or $M( \pm \mathrm{SD})$.

\begin{tabular}{|c|c|c|c|c|}
\hline Variables & Total: $N=32$ & NIHSS $\leq 6 ; N=15$ & NIHSS $>6 ; N=17$ & $\boldsymbol{p}$ \\
\hline Atheroma (mm) & $3.1 \pm 2.4$ & $4.5 \pm 2.3$ & $2.5 \pm 2.4$ & 0.03 \\
\hline Any atheroma & $21(65.6 \%)$ & $7 / 15(46.7 \%)$ & $14 / 17(82.4 \%)$ & 0.03 \\
\hline $\mathrm{AA}>4 \mathrm{~mm}$ as per literature & $15(46.9 \%)$ & $4(26.7 \%)$ & $11(64.7 \%)$ & 0.04 \\
\hline AA calcification & $18(56.3 \%)$ & $6(0.4 \%)$ & $12(70.5 \%)$ & 0.08 \\
\hline AA protrusion & $11(34.4 \%)$ & $3(20 \%)$ & $8(64.7 \%)$ & 0.15 \\
\hline AA ulceration & $11(34.4 \%)$ & $3(20 \%)$ & $8(64.7 \%)$ & 0.15 \\
\hline Age, years, $M \pm S D$ & $66.7 \pm 13.9$ & $62 \pm 17.7$ & $71 \pm 3.3$ & 0.09 \\
\hline \multicolumn{5}{|l|}{ ETHNICITY } \\
\hline White & $26(81 \%)$ & $11(73.3 \%)$ & $15(88.3 \%)$ & 0.28 \\
\hline Black & $6(19 \%)$ & $4(26.7 \%)$ & $2(11.8 \%)$ & \\
\hline \multicolumn{5}{|l|}{ GENDER } \\
\hline Female & $17(53 \%)$ & $8(53.3 \%)$ & $9(53 \%)$ & 0.99 \\
\hline Male & $15(47 \%)$ & $7(46.7 \%)$ & $8(47 \%)$ & \\
\hline Hypertension & $20(62.5 \%)$ & $7(46.7 \%)$ & $13(76.5 \%)$ & 0.08 \\
\hline Diabetes & $10(31.3 \%)$ & $3(20 \%)$ & $7(41.2 \%)$ & 0.27 \\
\hline Hyperlipidemia & 12 (37.5\%) & $3(20 \%)$ & $9(53 \%)$ & 0.08 \\
\hline CAD & $10(31.3 \%)$ & $1(6.7 \%)$ & $9(53 \%)$ & 0.01 \\
\hline PVD & $8(25 \%)$ & $3(20 \%)$ & $5(29.4 \%)$ & 0.70 \\
\hline Smoking & 10 (31.3\%) & $3(20 \%)$ & $7(42.2 \%)$ & 0.27 \\
\hline
\end{tabular}

CAD, coronary artery disease; PVD, peripheral vascular disease.

Table 2 | The final model with AA and propensity score adjustment (response: NIHSS (>6).

\begin{tabular}{|c|c|c|c|c|c|}
\hline Term & Estimate & Prob $>$ chi-square & Odds ratio & Lower $95 \%$ & Upper $95 \%$ \\
\hline Intercept & 1.91 & 0.59 & & & \\
\hline Age & -0.08 & 0.29 & 0.01 & 0.77 & 1.05 \\
\hline Propensity score [1] & 4.81 & 0.06 & 14.58 & 1.63 & 33.31 \\
\hline CT-AA [0-1] & 1.125 & 0.08 & 3.08 & 0.94 & 13.52 \\
\hline
\end{tabular}

a $p$-value of less than or equal to 0.5 (low power analysis due to sample size): age, any AA, HTN, DM, hyperlipidemia, and CAD (Table 3). Standard logistic regression analysis was performed using those variables to predict 3-month stroke outcome dichotomized at $\leq 1 \mathrm{mRS}$. A multiple logistic regression analysis model using the functional outcome as response and the effect of AA presence or absence, CAD, age, and PS was performed. PS was used as a weighted variable to adjust for all baseline variables that may be associated with AA. The final model including AA, age, $\mathrm{CAD}$, and PS revealed no association between 3-month mRS and AA presence ( $p=0.38$, OR 0.77, 95\% CI 0.4-11.31; see Table 4).

\section{DISCUSSION}

The principal goal of this investigation was to explore a potential association between "AA presence and thickness" and the severity of the neurological deficit at the onset of ischemic stroke. It has been difficult to correlate stroke severity with the extent of atherosclerotic change in pre-cerebral blood vessels (Timsit et al., 1993; Mitusch et al., 1997; Ferrari et al., 1999; Geraci and Weinberger, 2000; Paciaroni et al., 2000; Sen et al., 2000; Weinberger et al., 2000; Viguier et al., 2001; Matsumura et al., 2002).
Studies of carotid artery atherosclerosis did not find a simple correlation between stroke severity and degree of stenosis (Timsit et al., 1993; Mitusch et al., 1997; Ferrari et al., 1999; Geraci and Weinberger, 2000; Paciaroni et al., 2000; Sen et al., 2000; Weinberger et al., 2000; Viguier et al., 2001; Matsumura et al., 2002). One study found a correlation between worsened stroke severity and certain morphological features of carotid artery plaque, but not the degree of luminal narrowing (Paciaroni et al., 2000). There were higher incidences of death and major disabling stroke with "unstable" plaque, and features including ulceration, irregularities, and mobile protruding atheromas (Paciaroni et al., 2000). The possible correlation between AA and stroke severity has not been described previously. One study had shown a higher proportion of medium size subcortical strokes in patients with AA (Matsumura et al., 2002). Other studies have also shown increased risk of stroke severity when complex AA features are present (Weinberger et al., 2000; Zaidat et al., 2003). Our results indicate that AA presence on CGCT may be associated with worse initial stroke severity, the effect was less pronounced after adjusting for baseline covariates by applying a logistic regression model. 
Table 3 | Univariate analysis of the baseline characteristics and 3-month outcome ( $n$, \%, or $M( \pm \mathrm{SD})$.

\begin{tabular}{|c|c|c|c|c|}
\hline Variables & Total: $N=32$ & $m R S \leq 1 ; n=18$ & $m R S>1 ; n=14$ & $p$ \\
\hline $\mathrm{NIHSS}>6$ & 17/32 (53.1\%) & $5 / 18(27.8 \%)$ & $12 / 14(85.7 \%)$ & 0.002 \\
\hline Atheroma (mm) & $3.1 \pm 2.4$ & $3.2 \pm 2.5$ & $3.9 \pm 2.4$ & 0.4 \\
\hline Any atheroma & $21(65.6 \%)$ & $10(55.5 \%)$ & $11(78.6 \%)$ & 0.27 \\
\hline $\mathrm{AA}>4 \mathrm{~mm}$ & $15(46.9 \%)$ & $6(33.3 \%)$ & $9(64.3 \%)$ & 0.15 \\
\hline AA calcification & 18 (56.3\%) & $9(50 \%)$ & $9(64.3 \%)$ & 0.49 \\
\hline AA protrusion & $11(34.4 \%)$ & $5(27.8 \%)$ & $6(42.9 \%)$ & 0.47 \\
\hline AA ulceration & $11(34.4 \%)$ & $6(33.3 \%)$ & $5(35.7 \%)$ & 0.68 \\
\hline Age, years $(M \pm S D)$ & $66.7 \pm 13.9$ & $62.5 \pm 15.6$ & $72.5 \pm 8.6$ & 0.05 \\
\hline \multicolumn{5}{|l|}{ ETHNICITY } \\
\hline White & $26(81 \%)$ & $15(83.3 \%)$ & $11(78.6 \%)$ & 0.68 \\
\hline Black & $6(19 \%)$ & $3(16.7 \%)$ & $3(21.4 \%)$ & \\
\hline \multicolumn{5}{|l|}{ GENDER } \\
\hline Female & $17(53 \%)$ & $10(55.5 \%)$ & $7(50 \%)$ & 0.99 \\
\hline Male & $15(47 \%)$ & $8(44.4 \%)$ & $7(50 \%)$ & \\
\hline Hypertension & $20(62.5 \%)$ & $9(50 \%)$ & $11(78.6 \%)$ & 0.15 \\
\hline Diabetes & $10(31.3 \%)$ & $2(11.1 \%)$ & $8(57.1 \%)$ & 0.01 \\
\hline Hyperlipidemia & $12(37.5 \%)$ & $6(33.3 \%)$ & $6(42.9 \%)$ & 0.54 \\
\hline CAD & $10(31.3 \%)$ & $2(11.1 \%)$ & $8(57.1 \%)$ & 0.01 \\
\hline PVD & $8(25 \%)$ & $5(27.8 \%)$ & $3(21.4 \%)$ & 0.80 \\
\hline Smoking & $10(31.3 \%)$ & $5(27.8 \%)$ & $5(35.7 \%)$ & 0.71 \\
\hline
\end{tabular}

Table 4 | Logistic regression model with propensity score and AA: response functional outcome (modified Rankin Scale).

\begin{tabular}{|c|c|c|c|c|c|}
\hline Term & Estimate & Prob > chi-square & Odds ratio & Lower 95\% & Upper $95 \%$ \\
\hline Intercept & -1.59 & 0.62 & & & \\
\hline CT-AA & -0.25 & 0.38 & 0.77 & 0.4 & 11.31 \\
\hline CAD [0-1] & 1.28 & 0.08 & 12.83 & 1 & 22.13 \\
\hline Age & 0.032 & 0.54 & 7.38 & 0.93 & 11.17 \\
\hline Propensity score & 1.47 & 0.02 & 18.96 & 4.39 & 21.48 \\
\hline
\end{tabular}

To explore the correlation of AA with stroke outcome, we also looked at the association of AA with clinical outcome at 3-month after stroke (mRS), since the latter may bare a higher quality of life and health care impact than the initial stroke severity. Our findings showed that the plaque thickness was not associated with worse outcome at 3 months as was hypothesized, after adjusting for the baseline covariates using standard logistic regression and the PS $(p=0.38)$. This may be related to good patient recovery despite having suffered from severe stroke at presentation.

\section{REFERENCES}

Acarturk, E., Ozeren, A., and Sarica, Y. (1995). Detection of aortic plaques by transesophageal echocardiography in patients with ischemic stroke. Acta. Neurol. Scand. 92, 170-172.

Amanuallah, A. M., Artel, B. J., Grossman, L. B., Espioneza, A., and Chaudhry, F. A. (2002). Usefulness of complex atherosclerotic plaque in the ascending aorta and arch for predicting cardiovascular events. Am. J. Cardiol. 89, 1423-1426.
Cardenas, F. (1996). Protruding plaques of the thoracic aorta in the transesophageal echocardiogram: clinical and echographic characteristics. Rev. Med. Chil. 124, 1093-1099.

Castellanos, M., Serena, J., Segura, T., Perez-Ayuso,M.J.,Silva, Y., and Davalos, A. (2001). Atherosclerotic aortic arch plaques in cryptogenic stroke: a microembolic signal monitoring study. Eur. Neurol. 45, 145-150.

Chung, J. W., Park, J. H., Im, J. G., Chung, M. J., Han, M. C., and Ahn,

Our preliminary study is limited by the small sample size. Adjusting for confounding and interaction was not feasible due to the limited number of covariates that can be incorporated in the multivariate model. Applying a PS strengthened the study and allowed for adjustment for the observed but not the hidden bias.

In conclusion; the presence of AA may be associated with worse clinical severity at presentation. These findings need to be confirmed with TEE and spiral CT for stroke types and stroke severity in larger prospective stroke trials.

H. (1996). Spiral CT angiography of the thoracic aorta. Radiographics 16, 811-824.

Cohen, A., Tzourio, C., and Amarenco, P. (1997a). Evaluation of aortic atherosclerosis by transesophageal echocardiography. Prognostic implications. Arch. Mal. Coeur Vaiss. 90, 11-23.

Cohen, A., Tzourio, C., Bertrand, B., Chauvel, C., Bousser, M., Amarenco, P., On Behalf of the FAPS Investigators. (1997b). Aortic plaque morphology and vascular events: a follow-up study in patients with ischemic stroke. Circulation 96 , 3838-3841.

Costello, P., Ecker, C. P., Tello, R., and Hartnell, G. G. (1992). Assessment of the thoracic aorta by spiral CT. $A J R$ Am. J. Roentgenol. 158, 1127-1130.

Ferrari, E., Vidal, R., Chevallier, T., and Baudouy, M. (1999). Atherosclerosis of the thoracic aorta debris as a marker of poor prognosis: benefits of oral anticoagulants. J. Am. Coll. Cardiol. 33, 1371-1322. 
Flohr, T., Prokop, M., Becker, C., Schoepf, U. J., Kopp, A. F., White, R. D., Schaller, S., and Ohnesorge, B. (2002). A retrospectively ECGgated multislice spiral CT scan and reconstruction technique with suppression of heart pulsation artifacts for cardio-thoracic imaging with extended volume coverage. Eur. Radiol. 12, 1497-1503.

Fuchs, Y., Kachelriess, M., and Kalender, W. A. (2000). Technical advances in multi-slice spiral CT. Eur. J. Radiol. 36, 69-73.

Geraci, A., and Weinberger, J. (2000). Natural history of aortic arch atherosclerotic plaque. Neurology 54, 749-751.

Joffe, M. M., and Rosenbaum, P. R. (1999). Invited commentary: propensity scores. Am. J. Epidemiol. $150,327-333$.

Jones, E. F., Kalman, J. M., Calafiore, P., Tonkin, A. M., and Donnan, G. A. (1995). Proximal aortic atheroma. An independent risk factor for cerebral ischemia. Stroke 26, 218-224.

Matsumura, Y., Osaki, Y., Fukui, T., Yabe, T., Yamasaki, M., Hamashige, N., and Doi, Y. (2002). Protruding atherosclerotic aortic plaques and dyslipidemia: correlation to subtypes of ischemic stroke. Eur. J. Echocardiogr. $3,8-12$.

Mitusch, R., Doherty, C., Wucherpfennig, H., Memmesheimer, C., Tepe, C., Stierle, U., Kessler, C., and
Sheikhzadeh, A. (1997). Vascular events during follow-up patients with aortic arch atherosclerosis. Stroke 28, 36-39.

Paciaroni, M., Eliasziw, M., Sharpe, B. L., Kappelle, L. J., Chaturvedi, S., Meldrum, H., and Barnett, H. J. (2000). Long-term clinical and angiographic outcomes in symptomatic patients with $70 \%$ to $99 \%$ carotid artery stenosis. Stroke 31, 2037-2042.

Roos, J. E., Willmann, J. K., Weishaupt, D., Lachat, M., Marincek, B., and Hilfiker, P. R. (2002). Thoracic aorta: motion artifact reduction with retrospective and prospective electrocardiography-assisted multi-detector row CT. Radiology 222, 271-277.

Sen, S., Wu, K., McNamara, R., Lima, J., Piantadosi, S., and Oppenheimer, S. M. (2000). Distribution, severity and risk factors for aortic atherosclerosis in cerebral ischemia. Cerebrovasc. Dis. 10, 102-109.

The French Study of Aortic Plaques in Stroke Group. (1996). Atherosclerotic disease of the aortic arch as a risk factor for recurrent ischemic stroke. N. Engl. J. Med. 334, 1216-1221.

Timsit, S. G., Sacco, R. L., Mohr, J. P., Foulkes, M. A., Tatemichi, T. K., Wolf, P. A., Price, T. R., and Hier, D. B. (1993). Brain infarction severity differs according to cardiac or arterial embolic source. Neurology 43, 728-733.
Viguier, A., Pavy le Traon, A., Massabuau, P., Valton, L., and Larrue, V. (2001). Asymptomatic cerebral embolic signals in patients with acute cerebral ischemia and severe aortic arch atherosclerosis. J. Neurol. 248, 768-771.

Weinberger, J., Papamitsakis, N., Newfield, A., Godbold, J., and Goldman, M. (2000). Plaque morphology correlates with cerebrovascular symptoms in patients with complex aortic arch plaque. Arch. Neurol. 57, 81-84.

Zaidat, O. O., Eccher, M., Suarez, J. I., Sunshine, J., Tarr, R., and Gilkeson, R. C. (2002a). "The utility of cardiac cycle gated ultrafast contrasted computerized axial chest scan in the evaluation of patients with acute stroke and comparison with transesophageal echocardiography," in 54th Annual American Academy of Neurology Meeting, Denver, CO.

Zaidat, O. O., Suarez, J. I., Gilkeson, C. R., Sunshine, J., and Tarr, R. (2002b). The utility of ultrafast cardiac cycle gated and contrasted chest tomography in evaluation of patients with acute stroke and comparison with transesophageal echocardiography. Stroke 33, 358-359.

Zaidat, O. O., Suarez, J. I., Gilkeson, R. C., Sunshine, J. L., Tarr, R. W., Selman, W. R., and Landis, D. M. D. (2003). Initial ischemic stroke severity and 3 months outcome predicted by aortic atheroma location and morphology as detected by cardiac cycle gated and contrasted chest tomography. Neurology 60(Suppl. 1), A109-A110.

Conflict of Interest Statement: The authors declare that the research was conducted in the absence of any commercial or financial relationships that could be construed as a potential conflict of interest.

Received: 06 January 2011; accepted: 09 March 2011; published online: 21 March 2011.

Citation: Lazzaro MA, Zaidat OO, Issa MA, Gilkeson RC, Sunshine JL, Tarr RW, Husain S and Suarez JI (2011) Stroke severity predicted by aortic atheroma detected by ultra-fast and cardiac-gated chest tomography. Front. Neur. 2:18. doi: 10.3389/fneur.2011.00018

This article was submitted to Frontiers in Endovascular and Interventional Neurology, a specialty of Frontiers in Neurology.

Copyright (๑ 2011 Lazzaro, Zaidat, Issa, Gilkeson, Sunshine, Tarr, Husain and Suarez. This is an open-access article subject to an exclusive license agreement between the authors and the Articles Research Foundation, which permits unrestricted use, distribution, and reproduction in any medium, provided the original authors and source are credited. 